\title{
LADDER EPOCHS AND LADDER CHAIN OF A MARKOV RANDOM WALK WITH DISCRETE DRIVING CHAIN
}

\author{
BY GEROLD ALSMEYER
}

\begin{abstract}
Let $\left(M_{n}, S_{n}\right)_{n \geq 0}$ be a Markov random walk with positive recurrent driving chain $\left(M_{n}\right)_{n \geq 0}$ on the countable state space $\&$ with stationary distribution $\pi$. Suppose also that $\lim \sup _{n \rightarrow \infty} S_{n}=\infty$ almost surely, so that the walk has almost-sure finite strictly ascending ladder epochs $\sigma_{n}^{>}$. Recurrence properties of the ladder chain $\left(M_{\sigma_{n}^{>}}\right)_{n \geq 0}$ and a closely related excursion chain are studied. We give a necessary and sufficient condition for the recurrence of $\left(M_{\sigma_{n}}\right)_{n \geq 0}$ and further show that this chain is positive recurrent with stationary distribution $\pi^{>}$and $\mathbb{E}_{\pi^{>}}>\sigma_{1}^{>}<\infty$ if and only if an associated Markov random walk $\left(\hat{M}_{n}, \hat{S}_{n}\right)_{n \geq 0}$, obtained by time reversal and called the dual of $\left(M_{n}, S_{n}\right)_{n \geq 0}$, is positive divergent, i.e. $\hat{S}_{n} \rightarrow \infty$ almost surely. Simple expressions for $\pi^{>}$are also provided. Our arguments make use of coupling, Palm duality theory, and Wiener-Hopf factorization for Markov random walks with discrete driving chain.

Keywords: Markov-modulated sequence; Markov random walk; discrete Markov chain; ladder variable; ladder chain; excursion chain; stationary distribution; coupling; Palmduality; Wiener-Hopf factorization

2010 Mathematics Subject Classification: Primary 60J10

Secondary $60 \mathrm{~K} 15$
\end{abstract}

\section{Introduction}

Let $\left(M_{n}\right)_{n \geq 0}$ be an irreducible and positive recurrent Markov chain, defined on a probability space $(\Omega, \mathfrak{A}, \mathbb{P})$, with at most countable state space $\delta$, transition matrix $P=\left(p_{i j}\right)_{i, j \in \delta}$ and unique stationary distribution $\pi=\left(\pi_{i}\right)_{i \in s}$. Furthermore, let $\left(X_{n}\right)_{n \geq 0}$ be a sequence of realvalued random variables which are conditionally independent given $\left(M_{n}\right)_{n \geq 0}$ and

$$
\mathbb{P}\left(X_{n} \in \cdot \mid M_{0}, M_{1}, \ldots\right)=\mathbb{P}\left(X_{n} \in \cdot \mid M_{n-1}, M_{n}\right)=: F_{M_{n-1}, M_{n}} \quad \text { a.s. }
$$

for all $n \geq 1$. Equivalently, $\left(M_{n}, X_{n}\right)_{n \geq 0}$ is a Markov-modulated sequence when it is a temporally homogeneous Markov chain on $\delta \times \mathbb{R}$ whose transition kernel has the particular structure for which, for all $i, j \in \&, t, x \in \mathbb{R}$, and $n \geq 1$, and with $F_{i j}(t):=F_{i j}((-\infty, t])$,

$$
\begin{aligned}
Q((i, x),\{j\} \times(-\infty, t]): & \mathbb{P}\left(M_{n+1}=j, X_{n+1} \leq t \mid M_{n}=i, X_{n}=x\right) \\
& =\mathbb{P}\left(M_{n+1}=j, X_{n+1} \leq t \mid M_{n}=i\right) \\
& =p_{i j} F_{i j}(t) .
\end{aligned}
$$

As usual, we write $\mathbb{P}_{i}$ for $\mathbb{P}\left(\cdot \mid M_{0}=i\right), \mathbb{E}_{i}$ for expectations with respect to $\mathbb{P}_{i}$, and put $\mathbb{P}_{\lambda}:=\sum_{i \in \S} \lambda_{i} \mathbb{P}_{i}$ for any measure $\lambda=\left(\lambda_{i}\right)_{i \in \delta}$ on $\S$. Under $\mathbb{P}_{\pi},\left(M_{n}, X_{n}\right)_{n \geq 1}$ forms a stationary sequence and can therefore be extended to a doubly infinite sequence $\left(M_{n}, X_{n}\right)_{n \in \mathbb{Z}}$. Note that ' $\mathbb{P}_{\pi}$-a.s.', also written 'a.s.' hereafter, means $\mathbb{P}_{i}$-a.s. for all $i \in \delta$ because all $\pi_{i}$ are positive. In the doubly infinite setup, we further use $\mathbb{P}_{i, x}$ for $\mathbb{P}\left(\cdot \mid M_{0}=i, X_{0}=x\right)$ and let $\mathbb{P}_{v}$ have the 
obvious meaning for a measure $v$ on $\delta \times \mathbb{R}$. Finally, $\xi$ denotes the stationary distribution of $\left(M_{n}, X_{n}\right)_{n \in \mathbb{Z}}$.

Under the stated assumptions, the additive sequence $\left(S_{n}\right)_{n \geq 0}$, defined by $S_{0}:=0$ and $S_{n}:=\sum_{k=1}^{n} X_{k}$ for $n \geq 1$, as well as its bivariate extension $\left(M_{n}, S_{n}\right)_{n \geq 0}$ is called a Markov random walk (MRW) or a Markov-additive process, and $\left(M_{n}\right)_{n \geq 0}$ is its (discrete) driving chain. The MRW is called null-homologous (going back to [14]) if $X_{n}=g\left(M_{n}\right)-g\left(M_{n-1}\right)$ a.s. for all $n \geq 1$ and some function $g: s \rightarrow \mathbb{R}$, and then

$$
S_{n}=g\left(M_{n}\right)-g\left(M_{0}\right) \quad \text { a.s. }
$$

for all $n \geq 0$. Otherwise, the MRW is called nontrivial. When compared to ordinary random walks (RWs) with independent and identically distributed (i.i.d.) increments, the nullhomologous case, though comprising an infinite class of processes, corresponds to the trivial random walk with almost-sure zero increments and is ruled out in the following.

If $\left(S_{n}\right)_{n \geq 0}$ satisfies

$$
\limsup _{n \rightarrow \infty} S_{n}=\infty \quad \text { a.s. , }
$$

then the associated (strictly ascending) ladder height process is well defined, namely by $\left(S_{\sigma_{n}^{>}}\right)_{n \geq 0}$, where $\sigma_{0}^{>} \equiv 0$ and

$$
\sigma_{n}^{>}:=\inf \left\{k>\sigma_{n-1}^{>}: S_{k}>S_{\sigma_{n-1}^{>}}\right\}
$$

for $n \geq 1$ denote the corresponding ladder epochs. As usual, $\sigma_{n}^{>}:=\infty$ if $\sigma_{n-1}^{>}=\infty$ or the stopping condition is never met. Using the strong Markov property, it follows that

$$
\left(M_{n}^{>}, S_{n}^{>}\right)_{n \geq 0}:=\left(M_{\sigma_{n}}, S_{\sigma_{n}}\right)_{n \geq 0} \quad \text { and } \quad\left(M_{n}^{>}, \sigma_{n}^{>}\right)_{n \geq 0}
$$

form again MRWs. Their common driving chain $\left(M_{n}^{>}\right)_{n \geq 0}$ is called the ladder chain hereafter. Furthermore, defining $\vartheta_{0}:=0$ and $\vartheta_{n}:=\inf \left\{k>\vartheta_{n-1}: X_{k} \neq 0\right\}$ for $n \geq 1$, the sequence $\left(M_{\vartheta_{n}}, S_{\vartheta_{n}}\right)_{n \geq 0}$ forms another MRW whose driving chain is readily seen to be positive recurrent. Moreover, this MRW has almost-sure nonzero increments $S_{\vartheta_{n}}-S_{\vartheta_{n-1}}$ and the same ladder chain as $\left(M_{n}, S_{n}\right)_{n \geq 0}$ because $X_{\sigma_{n}^{>}}>0$ for all $n \geq 1$. Therefore, without loss of generality, we make the standing assumption that $\left(M_{n}, S_{n}\right)_{n \geq 0}$ itself has almost-sure nonzero increments, i.e.

$$
\mathbb{P}_{\pi}\left(X_{1}=0\right)=0 .
$$

This assumption enables us to avoid some technical issues regarding the excursion chain to be introduced in Subsection 2.2.

The main concern of this paper is to provide necessary and sufficient conditions for the recurrence and positive recurrence of $\left(M_{n}^{>}\right)_{n \geq 0}$ and a closely related augmentation $\left(M_{n}, E_{n}\right)_{n \geq 0}$ of the driving chain, introduced in Subsection 2.2 and which keeps track of the excursions of the random walk between the ladder epochs. Our results include information on the stationary distribution $\pi^{>}$, say, of $\left(M_{n}^{>}\right)_{n \geq 0}$ in terms of $\pi$. For the case of particular interest (at least in applied probability) when the stationary drift $\mu:=\mathbb{E}_{\pi} X_{1}$ exists finite and is positive (implying that $n^{-1} S_{n} \rightarrow \mu$ a.s. by Birkhoff's ergodic theorem and thus also (1.2)), the positive recurrence of $\left(M_{n}^{>}\right)_{n \geq 0}$ along with other properties of $\left(M_{n}^{>}, S_{n}^{>}, \sigma_{n}^{>}\right)_{n \geq 0}$ has already been proved in [1], even allowing the state space $\&$ to be continuous. On the other hand, the arguments given there are rather technical owing to the more complicated renewal structure when $\&$ is uncountable. We return briefly to this issue at the end of this paper. Here we draw on Palm calculus and WienerHopf factorization in combination with some fluctuation-theoretic properties of MRWs recently 
derived in [4] and shortly summarized in the next section. Apart from a paper by Fuh and Lai [12], where moments of ladder heights and first passage times for MRWs are studied, and some weakly related work on the Wiener-Hopf factorization for Markov-additive processes by Arjas and Speed [5], [6], Presman [17], and Asmussen [7], we are not aware of any work giving a systematic treatment of ladder variables and recurrence aspects of the associated ladder chain in the Markov-modulated setup. On the other hand, there is a large number of contributions dealing with other aspects of MRWs; see, e.g. the old surveys by Çinlar [9], [10], the monographs by Korolyuk and Turbin [13] and Asmussen [8, Chapter XI], and the references therein.

The recurrence of the ladder chain and related properties form an important ingredient when dealing with Markov renewal theory or, more generally, fluctuation-theoretic properties of a MRW $\left(M_{n}, S_{n}\right)_{n \geq 0}$, namely, they allow us to identify a certain subsequence $\left(S_{\tau_{n}(i)}\right)_{n \geq 0}$ of $\left(S_{n}^{>}\right)_{n \geq 0}$ and, thus, of $\left(S_{n}\right)_{n \geq 0}$; this subsequence is an ordinary renewal process (an RW with positive increments) because $M_{\tau_{n}>}(i)=i$ for all $n \geq 1$ and some $i \in \&$. Such embeddings are fundamental when we attempt to derive results of the aforementioned kind for MRWs by drawing on known results for ordinary RWs or renewal processes. For the case that $\left(M_{n}, S_{n}\right)_{n \geq 0}$ has positive stationary drift, we demonstrated this recently in [3] by showing that all fundamental Markov renewal theorems can be deduced with the help of such embeddings and the use of classical renewal theory.

\section{Preliminaries}

\subsection{Doubly infinite extension and fluctuation type}

Return to the doubly infinite sequence $\left(M_{n}, X_{n}\right)_{n \in \mathbb{Z}}$ under $\mathbb{P}_{\xi}$ and define the associated doubly infinite random walk $\left(S_{n}\right)_{n \in \mathbb{Z}}$ via

$$
S_{n}:= \begin{cases}\sum_{i=1}^{n} X_{i} & \text { if } n \geq 1, \\ 0 & \text { if } n=0, \\ -\sum_{i=n+1}^{0} X_{i} & \text { if } n \leq-1 ;\end{cases}
$$

then $S_{n}=S_{n-1}+X_{n}$ for all $n \in \mathbb{Z}$. Note that the forward sequence $\left(M_{n}, X_{n}\right)_{n \in \mathbb{Z}}$ is a stationary Markov chain with transition kernel $Q$ given by (1.1), while the backward sequence $\left(\hat{M}_{n}, \hat{X}_{n}\right)_{n \in \mathbb{Z}}:=\left(M_{-n}, X_{-n+1}\right)_{n \in \mathbb{Z}}$ is a stationary Markov chain with the dual kernel $\hat{Q}$ given for all $i, j \in \S$ and $x, t \in \mathbb{R}$ by

$$
\hat{Q}((i, x),\{j\} \times(-\infty, t])=\frac{\pi_{j} p_{j i}}{\pi_{i}} F_{j i}(t) .
$$

We call $\left(\hat{M}_{n}, \hat{X}_{n}\right)_{n \in \mathbb{Z}}$ and $\left(\hat{M}_{n}\right)_{n \in \mathbb{Z}}$ the dual of $\left(M_{n}, X_{n}\right)_{n \in \mathbb{Z}}$ and $\left(M_{n}\right)_{n \in \mathbb{Z}}$, respectively. Accordingly, the MRW $\left(\hat{M}_{n}, \hat{S}_{n}\right)_{n \in \mathbb{Z}}$, where $\hat{S}_{n}$ has the obvious meaning, is called the dual of $\left(M_{n}, S_{n}\right)_{n \geq 0}$. Note that $\hat{S}_{n}=-S_{-n}$ for $n \in \mathbb{Z}$.

Our earlier paper [4] shows that a nontrivial MRW $\left(M_{n}, S_{n}\right)_{n \geq 0}$ exhibits the same fluctuationtype trichotomy as an ordinary RW, meaning that exactly one of the following three alternatives occurs [4, Proposition 4.4]:

Positive divergence: $\lim _{n \rightarrow \infty} S_{n}=\infty$ a.s.;

Negative divergence: $\lim _{n \rightarrow \infty} S_{n}=-\infty$ a.s.;

Oscillation: $\lim \inf _{n \rightarrow \infty} S_{n}=-\infty$ and $\lim _{\sup _{n \rightarrow \infty}} S_{n}=\infty$ a.s.

Naturally, the same holds true for the dual $\left(\hat{M}_{n}, \hat{S}_{n}\right)_{n \geq 0}$. Moreover, letting $\tau_{0}(i):=0$ and $\tau_{n}(i)$ denote the $n$th return epoch of $M$ to $i$, the fluctuation-type of the embedded ordinary RW 
$\left(S_{\tau_{n}(i)}\right)_{n \geq 0}$ is the same for all $i$ (solidarity) (see [4, Lemma 6.1]), and also $\mathbb{P}_{i}\left(S_{\tau(i)} \in \cdot\right)=$ $\mathbb{P}_{i}\left(\hat{S}_{\hat{\tau}(i)} \in \cdot\right)$ for any $i$, where $\hat{\tau}(i)$ has the obvious meaning. On the other hand and somewhat surprisingly, the fluctuation types of $\left(M_{n}, S_{n}\right)_{n \geq 0}$ and its dual need not be the same as for the embedded RWs $\left(S_{\tau_{n}(i)}\right)_{n \geq 0}$ when the latter is positive or negative divergent. In fact, it is possible in this case for $\left(M_{n}, S_{n}\right)_{n \geq 0}$ and/or $\left(\hat{M}_{n}, \hat{S}_{n}\right)_{n \geq 0}$ to be oscillating (see [4, Example 7.2] for an illustrative example). In particular, the positive divergence of $\left(M_{n}, S_{n}\right)_{n \geq 0}$ does not necessarily entail the positive divergence of $\left(\hat{M}_{n}, \hat{S}_{n}\right)_{n \geq 0}$, that is,

$$
\lim _{n \rightarrow \infty} \hat{S}_{n}=\lim _{n \rightarrow \infty}-S_{-n}=\infty \quad \text { a.s. }
$$

can fail. On the other hand, $\left(M_{n}, S_{n}\right)_{n \geq 0}$ shares the fluctuation type with its dual and all its embedded RWs $\left(S_{\tau_{n}(i)}\right)_{n \geq 0}$ (and then we call it a solidary MRW) whenever

- $\&$ is finite; or

- the stationary drift $\mu=\mathbb{E}_{\pi} X_{1}$ is finite, because then

$$
\lim _{n \rightarrow \infty} n^{-1} S_{n}=\lim _{n \rightarrow \infty} n^{-1} \hat{S}_{n}=\mu \quad \text { a.s.; or }
$$

- the embedded RWs $\left(S_{\tau_{n}(i)}\right)_{n \geq 0}$ are oscillating.

If (2.2) is assumed then, with probability 1 , there is a doubly infinite sequence $\left(\sigma_{n}\right)_{n \in \mathbb{Z}}$ of ladder epochs for $\left(M_{n}, S_{n}\right)_{n \in \mathbb{Z}}$, determined through $\cdots<\sigma_{-1}<\sigma_{0} \leq 0<\sigma_{1}<\sigma_{2}<\cdots$ and $S_{\sigma_{n}}>\sup _{j<\sigma_{n}} S_{j}$ for all $n \in \mathbb{Z}$. In particular,

$$
\sigma_{1}:=\inf \left\{k \geq 1: S_{k}>\sup _{j<k} S_{j}\right\}, \quad \sigma_{0}:=\sup \left\{k \leq 0: S_{k}>\sup _{j<k} S_{j}\right\} .
$$

The reader should note that the sequences $\left(\sigma_{n}\right)_{n \geq 1}$ and $\left(\sigma_{n}^{>}\right)_{n \geq 1}$ are generally different although they have the same recursive structure (see (1.3)):

$$
\sigma_{n}=\inf \left\{k>\sigma_{n-1}: S_{k}-S_{\sigma_{n-1}}>0\right\} .
$$

The sequence $\left(\sigma_{n}\right)_{n \in \mathbb{Z}}$ enables us to provide a Palm-theoretic description of the stationary distribution of the ladder chain in the positive recurrent case (see (3.3) in Theorem 3.2).

Below we also need the sequence of dual weakly descending ladder epochs

$$
\hat{\sigma}_{n}^{\leqslant}:=\inf \left\{k>\hat{\sigma}_{n-1}^{\leqslant}: \hat{S}_{k} \leq \hat{S}_{\hat{\sigma}_{n-1}^{\leqslant}}\right\}
$$

for $n \geq 1$, where $\hat{\sigma}_{0}^{\leqslant}:=0$ and as usual inf $\varnothing:=\infty$.

\subsection{The excursion chain}

Define $S_{n}^{*}:=\max _{0 \leq k \leq n} S_{k}$ for $n \geq 0$, and let

$$
E_{n}:=S_{n}^{*}-S_{n}
$$

denote the excursion of $S_{n}$ from its current maximum at time $n$. Then $E_{0}=0$ and

$$
E_{n}=\left(E_{n-1}-X_{n}\right)^{+}
$$

for $n \geq 1$. If $\sigma_{k-1}^{>}<n<\sigma_{k}^{>}$for $k \in \mathbb{N}$, we can also write $E_{n}=-\sum_{l=\sigma_{k-1}^{>}+1}^{n} X_{l}$.

It is readily seen that $\left(M_{n}, E_{n}\right)_{n \geq 0}$ forms a temporally homogeneous Markov chain on $\delta \times[0, \infty)$, called the excursion chain hereafter. Recall our standing assumption (1.4), namely, 
$\mathbb{P}_{\pi}\left(X_{1}=0\right)=0$; then an excursion chain is defined on the ladder epochs because the latter are the successive hitting times of $\delta \times\{0\}$ for $\left(M_{n}, E_{n}\right)_{n \geq 0}$. If $E_{0}>0$, we put

$$
E_{n}:=E_{0}+S_{n}^{*}-S_{n} .
$$

Recall that $\left(M_{n}, E_{n}\right)_{n \geq 0}$ is called Harris recurrent or a Harris chain if there exist both

- a $\sigma$-finite measure $\varphi$ on $\& \times[0, \infty)$, called an irreducibility measure, such that any $\varphi$-positive subset $B$ of $\delta \times[0, \infty)$ is visited infinitely often (i.o.), i.e.

$$
\mathbf{P}_{i, e}\left(\left(M_{n}, E_{n}\right) \in B \text { i.o. }\right)=1 \text { for all }(i, e) \in \& \times[0, \infty),
$$

where $\mathbf{P}_{i, e}:=\mathbb{P}\left(\cdot \mid M_{0}=i, E_{0}=e\right)$; and

- a $\varphi$-positive set $C$, called a small set by Meyn and Tweedie [15, Section 5.2] and a regeneration set by Asmussen [8, p. 198], such that, for some $m \in \mathbb{N}$, some $\alpha \in(0,1]$, and a probability measure $\phi$ with $\phi(C)=1$,

$$
\mathbf{P}_{i, e}\left(\left(M_{m}, E_{m}\right) \in \cdot \cap C\right) \geq \alpha \phi(\cdot) .
$$

When these two conditions are met, $\left(M_{n}, E_{n}\right)_{n \geq 0}$ possesses an essentially unique, $\sigma$-finite stationary measure $\Lambda$, say, which is then also an irreducibility measure and assumed to be normalized if finite. In the latter case, the chain is called positive Harris recurrent or a positive Harris chain and $\Lambda$ is its unique stationary distribution. In the following, recurrence or positive recurrence of the excursion chain is always understood in the sense of Harris without being further mentioned. Some rather straightforward, but useful facts in connection with $\left(M_{n}, E_{n}\right)_{n \geq 0}$ and its ladder chain are summarized in the following lemma.

Lemma 2.1. (a) The excursion chain $\left(\left(M_{n}, E_{n}\right), E_{n}\right)_{n \geq 0}$ is a null-homologous MRW.

(b) The sequence $\left(\left(M_{n}, E_{n}\right), S_{n}^{*}\right)_{n \geq 0}$ is a MRW.

(c) The ladder chain $\left(M_{n}^{>}\right)_{n \geq 0}$ is recurrent if and only if $\left(M_{n}, E_{n}\right)_{n \geq 0}$ is recurrent.

(d) If $\left(M_{n}, E_{n}\right)_{n \geq 0}$ is recurrent with stationary measure $\Lambda$ satisfying $\Lambda(\& \times\{0\})<\infty$, then $\left(M_{n}^{>}\right)_{n \geq 0}$ is positive recurrent with stationary probabilities

$$
\pi_{i}^{>}=\Lambda(\{i\} \times\{0\}) / \Lambda(\& \times\{0\}), \quad i \in \& .
$$

Proof. For (a), put $g(i, e):=e$ for all $(i, e) \in \delta \times[0, \infty)$ and note that, for $n \geq 1$,

$$
E_{n}-E_{n-1}=g\left(M_{n}, E_{n}\right)-g\left(M_{n-1}, E_{n-1}\right) .
$$

Assertion (b) follows from $S_{n}^{*}-S_{n-1}^{*}=X_{n}+E_{n}-E_{n-1}$ for all $n \geq 1$, and we obtain (c) by observing that $M^{>}$visits a state $i$ infinitely often if and only if $\left(M_{n}, E_{n}\right)_{n \geq 0}$ visits the state $(i, 0)$ infinitely often, in which case $\{(i, 0)\}$ forms a regeneration set. The stationary measure $\Lambda$ of $\left(M_{n}, E_{n}\right)_{n \geq 0}$ is then obtained as

$$
\Lambda(B)=\frac{1}{\mathfrak{m}_{i}} \mathbf{E}_{i, 0}\left(\sum_{n=1}^{\tau(i, 0)} \mathbf{1}_{B}\left(M_{n}, E_{n}\right)\right)
$$

for all measurable $B \subset \& \times[0, \infty)$, where $\tau(i, 0):=\inf \left\{n \geq 1:\left(M_{n}, E_{n}\right)=(i, 0)\right\}$ and

$$
\mathfrak{m}_{i}:= \begin{cases}\mathbf{E}_{i, 0} \tau(i, 0) & \text { if this mean is finite } \\ 1 & \text { otherwise. }\end{cases}
$$


Finally, if $\Lambda(8 \times[0, \infty))=1$ (positive recurrence), then $\pi^{>}$as stated in the lemma is a stationary distribution for the chain $\left(M_{n}^{>}\right)_{n \geq 0}$, thus proving its positive recurrence.

In view of parts (c) and (d) of this lemma, it is natural to study recurrence properties of $\left(M_{n}^{>}\right)_{n \geq 0}$ and $\left(M_{n}, E_{n}\right)_{n \geq 0}$ together whenever convenient. This paper has two main results. Theorem 3.1 shows, under the minimal requirement (1.2) for the almost-sure finiteness of the ladder epochs, that $\left(M_{n}^{>}\right)_{n \geq 0}$ is either transient or recurrent on a unique irreducibility class $s^{>} \subset \&$ (this is defined in (3.1)). It also provides a necessary and sufficient condition for recurrence in terms of the dual weakly descending ladder epoch $\hat{\sigma}_{1}^{\leqslant}=\inf \left\{n: \hat{S}_{n} \leq 0\right\}$ and, in particular, shows the sufficiency of the condition

$$
\limsup _{n \rightarrow \infty} \hat{S}_{n}=\infty \quad \text { a.s. }
$$

Our second result, Theorem 3.2, provides necessary and sufficient conditions for the positive recurrence of $\left(M_{n}, E_{n}\right)_{n \geq 0}$. These include both the positive recurrence of $\left(M_{n}^{>}\right)_{n \geq 0}$ and the finiteness condition $\mathbb{E}_{\pi}>\sigma_{1}^{>}<\infty$. With regard to applications, this covers what are presumably the most important cases (including the case when the stationary drift $\mathbb{E}_{\pi} X_{1}$ is positive), but leaves open the far more difficult problem of a corresponding characterization of the situation when $\mathbb{E}_{\pi}>\sigma_{1}^{>}=\infty$ (see Section 6 for some further comments).

\subsection{Wiener-Hopf factorization}

We derive an interesting formula for the stationary distribution of the ladder chain (see (3.4) of Theorem 3.2) with the help of the Wiener-Hopf factorization for MRWs. Here we refer to [7] and [8, p. 314ff] in recalling some necessary facts about this factorization.

Writing $\left(\hat{M}_{n}^{\leqslant}, \hat{S}_{n}^{\leqslant}\right):=\left(\hat{M}_{\hat{\sigma}_{n}} \leqslant, \hat{S}_{\hat{\sigma}_{n}} \leqslant\right)$, define the matrices

$$
G:=\left(G_{i j}\right)_{i, j \in \mathcal{S}}, \quad G^{>}:=\left(G_{i j}^{>}\right)_{i, j \in \mathcal{S}}, \quad \hat{G}^{\leqslant}:=\left(\hat{G}_{i j}^{\leqslant}\right)_{i, j \in \mathcal{S}}, \quad{ }^{\star} G^{\leqslant}:=\left({ }^{\star} G_{i j}^{\leqslant}\right)_{i, j \in \mathcal{S}},
$$

whose measure-valued elements are given by

$$
\begin{aligned}
G_{i j} & :=p_{i j} F_{i j}=\mathbb{P}_{i}\left(M_{1}=j, X_{1} \in \cdot\right), \\
G_{i j}^{>} & :=\mathbb{P}_{i}\left(M_{1}^{>}=j, S_{1}^{>} \in \cdot, \sigma_{1}^{>}<\infty\right), \\
\hat{G}_{i j}^{\leqslant} & :=\mathbb{P}_{i}\left(\hat{M}_{1}^{\leqslant}=j, \hat{S}_{1}^{\leqslant} \in \cdot \hat{\sigma}_{1}^{\leqslant}<\infty\right), \\
{ }^{\star} G_{i j}^{\leqslant} & :=\frac{\pi_{j}}{\pi_{i}} \hat{G}_{j i}^{\leqslant} .
\end{aligned}
$$

The convolution of matrices $A=\left(A_{i j}\right)_{i, j \in \delta}$ and $B=\left(B_{i j}\right)_{i, j \in \delta}$ with measure-valued entries is defined in the usual way by replacing ordinary multiplication by the convolution of measures; thus, $A * B=\left(\sum_{k \in \S} A_{i k} * B_{k j}\right)_{i, j \in s}$. The following result, stated here for reference, provides the Wiener-Hopffactorization of an MRW with discrete driving chain and is at [7, Theorem 4.1] for finite $\delta$ and at [16, Theorem 10] for countable $\delta$ (see also [12, Equation (4.7)]). For a more abstract approach to Wiener-Hopf factorizations of MRWs, see the work of Presman [17] and Arjas and Speed [5], [6].

Proposition 2.1. Let $\left(M_{n}, S_{n}\right)_{n \geq 0}$ be a MRW with positive recurrent discrete driving chain $\left(M_{n}\right)_{n \geq 0}$. Then

$$
\delta_{0} I-G=\left(\delta_{0} I-{ }^{\star} G^{\lessgtr}\right) *\left(\delta_{0} I-G^{>}\right)
$$

or, equivalently,

$$
G={ }^{\star} G^{\leqslant}+G^{>}-{ }^{\star} G^{\leqslant} * G^{>},
$$

where I denotes the identity matrix on 8 . 
We need the following lemma in which $\|v\|$ denotes the total mass of a measure $v$.

Lemma 2.2. Suppose that $\left(\hat{M}_{n}, \hat{S}_{n}\right)_{n \geq 0}$ is positive divergent so that (2.2) holds. Then the matrix $\left\|\hat{G}^{\leqslant}\right\|:=\left(\left\|\hat{G}_{i j}^{\leqslant}\right\|\right)_{i, j \in \mathcal{S}}$ is truly substochastic, i.e.

$$
\sum_{j \in \delta}\left\|\hat{G}_{i j}^{\leqslant}\right\| \leq 1
$$

for all $i \in \&$ with strict inequality for at least one $i$. Furthermore,

$$
\sum_{i \in \delta} \pi_{i}\left\|^{\star} G_{i j}^{\leqslant}\right\|=\pi_{j} \mathbb{P}_{j}\left(\hat{\sigma}_{1}^{\leqslant}<\infty\right) \leq \pi_{j}
$$

for all $j \in \delta$ with strict inequality for at least one $j$.

Proof. From the definition of the $\hat{G}_{i j}^{\leqslant}$, we have, for all $i \in \&$,

$$
\sum_{j \in \delta}\left\|\hat{G}_{i j}^{\leqslant}\right\|=\sum_{j \in \mathcal{S}} \mathbb{P}_{i}\left(\hat{M}_{1}^{\leqslant}=j, \hat{\sigma}_{1}^{\leqslant}<\infty\right)=\mathbb{P}_{i}\left(\hat{\sigma}_{1}^{\leqslant}<\infty\right) \leq 1 .
$$

Moreover, strict inequality must hold for at least one $i$, for otherwise $\mathbb{P}_{\pi}\left(\hat{\sigma}_{1}^{\leqslant}<\infty\right)=1$ would follow and then inductively $\mathbb{P}_{\pi}\left(\hat{\sigma}_{n}^{\leqslant}<\infty\right)=1$ for all $n \in \mathbb{N}$, i.e.

$$
\mathbb{P}_{\pi}\left(\hat{S}_{n} \leq 0 \text { i.o. }\right)=1
$$

which is impossible by the positive divergence of $\left(\hat{S}_{n}\right)_{n \geq 0}$.

To prove (2.6), note that

$$
\sum_{i \in \mathcal{S}} \pi_{i}\left\|^{\star} G_{i j}^{\leqslant}\right\|=\sum_{i \in \mathcal{S}} \pi_{j}\left\|\hat{G}_{j i}^{\leqslant}\right\|=\pi_{j} \sum_{i \in \mathcal{S}} \mathbb{P}_{j}\left(\hat{M}_{1}^{\leqslant}=i, \hat{\sigma}_{1}^{\leqslant}<\infty\right)=\pi_{j} \mathbb{P}_{j}\left(\hat{\sigma}_{1}^{\leqslant}<\infty\right),
$$

and, by an analogous argument showing strict inequality for some $i$, this value must be less than $\pi_{j}$ for at least one $j$ when $\left(\hat{S}_{n}\right)_{n \geq 0}$ is positive divergent.

\section{Main results}

In presenting our main results, we assume throughout that there is a given MRW $\left(M_{n}, S_{n}\right)_{n \geq 0}$ which has a positive recurrent driving chain $\left(M_{n}\right)_{n \geq 0}$ on the countable state space $\delta$, with transition matrix $P=\left(p_{i j}\right)_{i, j \in s}$ and stationary distribution $\pi$, and the minimal condition (1.2) for the existence of the ladder chain $\left(M_{n}^{>}\right)_{n \geq 0}$ is satisfied.

Theorem 3.1. Under the stated assumptions, define

$$
s^{>}:=\left\{j \in s: \sup _{k \geq 1} \mathbb{P}_{i}\left(M_{k}^{>}=j\right)>0 \text { for all } i \in s\right\}
$$

and $\tau^{>}\left(\delta^{>}\right):=\inf \left\{n \geq 1: M_{n}^{>} \in s^{>}\right\}$. Then

(a) the set $\varsigma^{>}$is either empty or the unique irreducibility class of $\left(M_{n}^{>}\right)_{n \geq 0}$, and in the latter case

$$
\mathbb{P}_{i}\left(\tau^{>}\left(\delta^{>}\right)<\infty\right)=1 \text { for all } i \in \delta
$$

(consequently, $\left(M_{n}^{>}\right)_{n \geq 0}$ is either transient or recurrent on $\delta^{>}$); and

(b) recurrence (and, thus, $\delta^{>} \neq \varnothing$ ) implies (2.4), i.e. $\lim \sup _{n \rightarrow \infty} \hat{S}_{n}=\infty$ a.s., and is equivalent to $\mathbb{E}_{i} \hat{\sigma}_{1}^{\leqslant}=\infty$ for at least one $i \in \delta$, in which case

$$
s^{>}=\left\{i \in s: \mathbb{E}_{i} \hat{\sigma}_{1}^{\leqslant}=\infty\right\}
$$


Remark 3.1. Obviously, $\delta^{>} \subset\left\{j \in \delta: p_{i j} F_{i j}((0, \infty))>0\right.$ for some $\left.i \in \delta\right\}$, and it equals the whole state space $\&$ whenever $p_{i j} F_{i j}((0, \infty))>0$ for all $i, j \in \&$.

Remark 3.2. The proof of Theorem 3.1 is given in Section 4, and shows in fact that all assertions remain valid if the driving chain $\left(M_{n}\right)_{n \geq 0}$ is null recurrent and $\pi$ is its essentially unique stationary measure. To some extent, this is also the case for the next result, but we refrain from going into further detail.

Theorem 3.2. Under the stated assumptions, the assertions below are equivalent.

(a) The excursion chain $\left(M_{n}, E_{n}\right)_{n \geq 0}$ is positive recurrent.

(b) The ladder chain $\left(M_{n}^{>}\right)_{n \geq 0}$ is positive recurrent with stationary distribution $\pi^{>}$, say, and $\mathbb{E}_{\pi}>\sigma_{1}^{>}<\infty$.

(c) The bivariate chain $\left(M_{n}^{>}, \sigma_{n+1}^{>}-\sigma_{n}^{>}\right)_{n \geq 0}$ is positive recurrent with stationary distribution $v$, say, and $\mathbb{E}_{\nu} \sigma_{1}^{>}<\infty$.

(d) The dual MRW $\left(\hat{M}_{n}, \hat{S}_{n}\right)_{n \geq 0}$ is positive divergent, i.e., (2.2) holds.

(e) The embedded $R W s\left(S_{\tau_{n}(i)}\right)_{n \geq 0}$ are positive divergent and $\mathbb{E}_{i} J_{i}\left(D^{i}\right)<\infty$ for some (and then all) $i \in \mathcal{S}$, where $D^{i}:=\max _{1 \leq k \leq \tau(i)} S_{k}^{+}$and $J_{i}$ is defined by

$$
J_{i}(0):=1 \quad \text { and } \quad J_{i}(x):=\left\{\begin{array}{ll}
\frac{x}{\mathbb{E}_{i}\left(S_{\tau(i)}^{+} \wedge x\right)} & \text { if } \mathbb{P}_{i}\left(S_{\tau(i)}>0\right)>0, \\
x & \text { otherwise, }
\end{array} \text { for } x>0 .\right.
$$

(f) $\mathbb{P}_{i}\left(\hat{\sigma}_{1}^{\leqslant}=\infty\right)>0$ for some (and then all) $i \in 8^{>}$.

When these assertions hold, the stationary distribution $\pi^{>}=\left(\pi_{i}^{>}\right)_{i \in \delta}$ is given by

$$
\pi_{i}^{>}:=\frac{1}{c} \mathbb{E}_{\xi}\left(\frac{1}{\sigma_{1}-\sigma_{0}} \mathbf{1}_{\left\{M_{\sigma_{0}}=i\right\}}\right)=\frac{1}{c} \mathbb{P}_{\xi}\left(M_{0}=i, \sigma_{0}=0\right),
$$

and has $\pi$-density $f(i)=c^{-1} \mathbb{P}_{\xi}\left(\sigma_{0}=0 \mid M_{0}=i\right)$ for $i \in 8$, where $\xi$ denotes the stationary law of $\left(M_{n}, X_{n}\right)_{n \in \mathbb{Z}}(c f$. below (1)) and

$$
c:=\mathbb{E}_{\xi}\left(\frac{1}{\sigma_{1}-\sigma_{0}}\right)=\mathbb{P}_{\xi}\left(\sigma_{0}=0\right) \in(0,1]
$$

Furthermore,

$$
\pi_{i}^{>}=\frac{1}{c} \pi_{i} \mathbb{P}_{i}\left(\hat{\sigma}_{1}^{\leqslant}=\infty\right)=\frac{1}{c} \mathbb{P}_{\pi}\left(\hat{M}_{0}^{\leqslant}=i, \hat{\sigma}_{1}^{\leqslant}=\infty\right)
$$

for all $i \in$ \&, implying that

$$
c=\mathbb{P}_{\pi}\left(\hat{\sigma}_{1}^{\leqslant}=\infty\right) .
$$

Finally, the stationary law $v=\left(v_{i, m}\right)_{(i, m) \in \delta \times \mathbb{N}}$ of $\left(M_{n}^{>}, \sigma_{n+1}^{>}-\sigma_{n}^{>}\right)_{n \geq 0}$ is given by

$$
v_{i, m}=\frac{1}{c m} \mathbb{P}_{\xi}\left(M_{\sigma_{0}}=i, \sigma_{1}-\sigma_{0}=m\right)
$$

in particular,

$$
\mathbb{P}_{\nu}\left(\sigma_{1}^{>}=m\right)=\mathbb{P}_{\pi}>\left(\sigma_{1}^{>}=m\right)=\sum_{i \in \delta} v_{i, m}=\frac{\mathbb{P}_{\xi}\left(\sigma_{1}-\sigma_{0}=m\right)}{c m}
$$

and

$$
\mathbb{E}_{\nu} \sigma_{1}^{>}=\mathbb{E}_{\pi}>\sigma_{1}^{>}=\frac{1}{\mathbb{P}_{\pi}\left(\hat{\sigma}_{1}^{\leqslant}=\infty\right)}
$$


Remark 3.3. Since, for any $i \in \delta, \pi_{i}^{>} \mathbb{E}_{i} \sigma_{1}^{>} \leq \mathbb{E}_{\pi^{>}} \sigma_{1}^{>}$and $\pi_{i}^{>}=c^{-1} \pi_{i} \mathbb{P}_{i}\left(\hat{\sigma}_{1}^{\leqslant}=\infty\right)$, (3.7) also shows that

$$
\mathbb{E}_{i} \sigma_{1}^{>} \leq \frac{1}{\pi_{i} \mathbb{P}_{i}\left(\hat{\sigma}_{1}^{\leqslant}=\infty\right)}
$$

for all $i \in \delta$, where the right-hand side is finite only for $i \in \mathcal{8}^{>}$.

Remark 3.4. In the case that $\left(M_{n}, S_{n}\right)_{n \geq 0}$ has stationary drift $0<\mu<\infty$ and, thus, also (2.2) holds (by solidarity), Theorem 3.2(c) can be viewed as a specialization of a similar result stated without proof by Lalley [14, Section 4B] for general random walks with integrable stationary increments.

A direct proof of Theorem 3.2 is given in Section 5, but the existence of $\pi^{>}$can also be deduced by drawing on a more general duality result from Palm calculus as described in the monographs of Thorisson [19, Chapter 8] and Sigman [18]. We refer to Remark 5.1 for a sketch of details, while in Remark 5.2 we indicate how the stationarity of $\pi^{>}$, when defined by (3.4), can also be verified with the help of the Wiener-Hopf factorization for MRWs.

\section{Proof of Theorem 3.1}

It is clear from the definition that $\delta^{>}$, if nonempty, forms the unique irreducibility class for $M^{>}$, so (a) holds. It thus remains to show (3.2); we do this in a coupling argument below.

Suppose that $\delta^{>}$is nonempty. On a possibly enlarged probability space with underlying probability measure $\mathbb{P}$, let $\left(M_{n}^{\prime}, X_{n}^{\prime}\right)_{n \geq 0}$ and $\left(M_{n}^{\prime \prime}, X_{n}^{\prime \prime}\right)_{n \geq 0}$ be two Markov-modulated sequences with the same transition kernel as $\left(M_{n}, X_{n}\right)_{n \geq 0}$ and initial condition $\left(M_{0}^{\prime}, M_{0}^{\prime \prime}\right)=(i, j)$ for arbitrarily fixed $(i, j) \in \delta \times \delta^{>}$. As usual, we denote the associated RWs by $\left(S_{n}^{\prime}\right)_{n \geq 0}$ and $\left(S_{n}^{\prime \prime}\right)_{n \geq 0}$, and the corresponding strictly ascending ladder epochs by $\sigma_{n}^{\prime}$ and $\sigma_{n}^{\prime \prime}$, respectively, where $\sigma_{0}^{\prime}=\sigma_{0}^{\prime \prime}:=0$. Define $\tau:=\inf \left\{n \geq 0: M_{n}^{\prime}=j\right\}$, and couple the two MRWs through

$$
\left(M_{\tau+n}^{\prime}, X_{\tau+n}^{\prime}\right)=\left(M_{n}^{\prime \prime}, X_{n}^{\prime \prime}\right) \text { for all } n \geq 0 .
$$

In other words, the primed walk moves until its driving chain hits state $j \in \delta^{>}$for the first time and is then coupled to the double primed walk in the sense that both use the same driving chain and make identical jumps. Since $\delta^{>}$is an irreducibility class,

$$
\mathbb{P}\left(M_{\sigma_{n}^{\prime \prime}}^{\prime \prime} \in s^{>} \text {for all } n \geq 0\right)=\mathbb{P}_{j}\left(M_{n}^{>} \in s^{>} \text {for all } n \geq 0\right)=1 .
$$

Now observe that, if $\sigma_{\rho}^{\prime}$ denotes the first ladder epoch after $\tau$ of $\left(M_{n}^{\prime}, S_{n}^{\prime}\right)_{n \geq 0}, \operatorname{so} \rho=\inf \left\{n: \sigma_{n}^{\prime}>\right.$ $\tau\}$, then $\sigma_{\rho}^{\prime}=\sigma_{\eta}^{\prime \prime}$ for $\eta=\inf \left\{n \geq 0: S_{\sigma_{n}^{\prime \prime}}^{\prime \prime}>\max _{0 \leq k \leq \tau} S_{k}^{\prime}-S_{\tau}^{\prime}\right\}$ and, therefore,

$$
\left(\sigma_{\rho+n}^{\prime}, M_{\sigma_{\rho+n}^{\prime}}^{\prime}\right)=\left(\sigma_{\eta+n}^{\prime \prime}, M_{\sigma_{\eta+n}^{\prime \prime}}^{\prime \prime}\right) \text { for all } n \geq 0 .
$$

Consequently,

$$
\begin{aligned}
\mathbb{P}_{i}\left(\tau\left(\delta^{>}\right)<\infty\right) & =\mathbb{P}\left(M_{\sigma_{n}^{\prime}}^{\prime} \in \delta^{>} \text {eventually }\right) \\
& =\mathbb{P}\left(M_{\sigma_{\rho+n}^{\prime}}^{\prime} \in \delta^{>} \text {eventually }\right) \\
& =\mathbb{P}\left(M_{\sigma_{\eta+n}^{\prime \prime}}^{\prime \prime} \in s^{>} \text {eventually }\right) \\
& =\mathbb{P}\left(M_{\sigma_{n}^{\prime \prime}}^{\prime \prime} \in \delta^{>} \text {for all } n \geq 0\right) \\
& =1,
\end{aligned}
$$

and this is the desired conclusion. 
Lemma 4.1. For any $\operatorname{MRW}\left(M_{n}, S_{n}\right)_{n \geq 0}$,

$$
\pi_{i} \mathbb{E}_{i} \hat{\sigma}_{1}^{\leqslant}=\sum_{n \geq 0} \mathbb{P}_{\pi}\left(M_{n}^{>}=i, \sigma_{n}^{>}<\infty\right)
$$

Proof. We start from the simple observation that, for all $s \in(0,1)$,

$$
\pi_{i} \frac{1-\mathbb{E}_{i} s^{\hat{\sigma}_{1}}}{1-s}=\mathbb{E}_{\pi}\left(\mathbf{1}_{\left\{\hat{M}_{0}=i\right\}} \sum_{n=0}^{\hat{\sigma}_{1}^{\leqslant}-1} s^{n}\right) .
$$

Using the duality relation

$$
\mathbb{P}_{\pi}\left(\left(\hat{M}_{k}, \hat{S}_{k}\right)_{0 \leq k \leq n} \in \cdot\right)=\mathbb{P}_{\pi}\left(\left(M_{n-k}, S_{n}-S_{n-k}\right)_{0 \leq k \leq n} \in \cdot\right),
$$

we further obtain for the right-hand side

$$
\begin{aligned}
\mathbb{E}_{\pi}\left(\mathbf{1}_{\left\{\hat{M}_{0}=i\right\}} \sum_{n=0}^{\hat{\sigma}_{1}^{\leqslant}-1} s^{n}\right) & =\sum_{n \geq 0} s^{n} \mathbb{P}_{\pi}\left(\hat{M}_{0}=i, \hat{\sigma}_{1}^{\leqslant}>n\right) \\
& =\sum_{n \geq 0} s^{n} \mathbb{P}_{\pi}\left(\hat{M}_{0}=i, \hat{S}_{k}>0 \text { for } 1 \leq k \leq n\right) \\
& =\sum_{n \geq 0} s^{n} \mathbb{P}_{\pi}\left(M_{n}=i, S_{n}-S_{n-k}>0 \text { for } 1 \leq k \leq n\right) \\
& =\sum_{n \geq 0} s^{n} \sum_{m \geq 0} \mathbb{P}_{\pi}\left(M_{n}=i, \sigma_{m}^{>}=n\right) \\
& =\mathbb{E}_{\pi}\left(\sum_{m \geq 0} s^{\sigma_{m}^{>}} \mathbf{1}_{\left\{M_{m}^{>}=i, \sigma_{m}^{>}<\infty\right\}}\right) .
\end{aligned}
$$

The assertion of the lemma follows on letting $s \uparrow 1$.

We now complete the proof of Theorem 3.1(b). Note first that $\mathbb{E}_{i} \hat{\sigma}_{1}^{\leqslant}=\infty$ for some $i \in \&$ implies that the dual MRW cannot be negative divergent and, thus, $\lim _{n \rightarrow \infty} \sup _{n \rightarrow \infty} \hat{S}_{n}=\infty$ a.s. by [4, Theorem 6.1]. It therefore remains to show the asserted equivalence. It is a well-known fact that $i$ is a recurrent state for $\left(M_{n}^{>}\right)_{n \geq 0}$ if and only if $\sum_{n \geq 0} \mathbb{P}_{i}\left(M_{n}^{>}=i\right)=\infty$, which in turn holds true if and only if $\sum_{n \geq 0} \mathbb{P}_{\pi}\left(M_{n}^{>}=i\right)=\infty$ as the following argument shows. Put $\tau^{>}(i)=\inf \left\{n \geq 1: M_{n}^{>}=i\right\}$. Then

$$
\begin{aligned}
\pi_{i} \sum_{n \geq 0} \mathbb{P}_{i}\left(M_{n}^{>}=i\right) & \leq \sum_{n \geq 0} \mathbb{P}_{\pi}\left(M_{n}^{>}=i\right) \\
& =\left(\pi_{i}+\sum_{j \in S \backslash\{i\}} \pi_{j} \mathbb{P}_{j}\left(\tau^{>}(i)<\infty\right)\right) \sum_{n \geq 0} \mathbb{P}_{i}\left(M_{n}^{>}=i\right) \\
& \leq \sum_{n \geq 0} \mathbb{P}_{i}\left(M_{n}^{>}=i\right) .
\end{aligned}
$$

The assertion (b) now follows directly from Lemma 4.1. 


\section{Proof of Theorem 3.2}

(a) and (b) are equivalent. If $\left(M_{n}, E_{n}\right)_{n \geq 0}$ is positive recurrent with stationary distribution $\Lambda$, then, by Lemma $2.1(\mathrm{~d}),\left(M_{n}^{>}\right)_{n \geq 0}$ is also positive recurrent with stationary distribution $\pi^{>}=\Lambda(\cdot \times\{0\}) / \Lambda(\delta \times\{0\})$. This shows that (a) implies (b). For the converse, let $\mathbf{P}$ be such that $\left(M_{n}, E_{n}\right)_{n \geq 0}$ has initial distribution $\pi^{>} \otimes \delta_{0}$ under $\mathbf{P}$. Note that, under $\mathbf{P}$, the cycles

$$
\left(\sigma_{n+1}^{>}-\sigma_{n}^{>},\left(M_{k}, E_{k}\right)_{\sigma_{n}^{>}<k \leq \sigma_{n+1}^{>}}\right), \quad n=0,1, \ldots,
$$

constitute a stationary sequence of finite mean length $\mathbb{E}_{\pi}>\sigma_{1}^{>}=\mathbf{E} \sigma_{1}^{>}$. Defining

$$
\Lambda(A):=\frac{1}{\mathbf{E} \sigma_{1}^{>}} \mathbf{E}\left(\sum_{n=1}^{\sigma_{1}^{>}} \mathbf{1}_{A}\left(M_{n}, E_{n}\right)\right)
$$

for measurable $A \subset \delta \times[0, \infty)$, it is now readily verified that $\Lambda$ is a stationary distribution for $\left(M_{n}, E_{n}\right)_{n \geq 0}$, thus proving the asserted positive recurrence of this chain.

(b) and (c) are equivalent. This follows directly from the fact that $\left(M_{n}, \sigma_{n+1}^{>}-\sigma_{n}^{>}\right)_{n \geq 0}$ is a Markov-modulated sequence as defined at the beginning of the introduction.

(d) and (e) are equivalent. Here we use a result from fluctuation theory for MRWs as developed in [4]. Note first that, by a result due to Erickson [11], the positive divergence of the ordinary $\mathrm{RW}\left(S_{\tau_{n}(i)}\right)_{n \geq 0}$ is equivalent to

$$
\mathbb{E}_{i}\left(S_{\tau(i)}^{+} \wedge x\right)-\mathbb{E}_{i}\left(S_{\tau(i)}^{-} \wedge x\right)>0 \quad \text { for all sufficiently large } x>0
$$

and

$$
\mathbb{E}_{i} J_{i}\left(S_{\tau(i)}^{-}\right)<\infty
$$

By duality, we further have, under $\mathbb{P}_{i}$,

$$
\left(\hat{\tau}(i),\left(\hat{M}_{n}, \hat{S}_{n}\right)_{1 \leq n \leq \hat{\tau}(i)}\right) \stackrel{\mathrm{D}}{=}\left(\tau(i),\left(M_{\tau(i)-n}, S_{\tau(i)}-S_{n}\right)_{1 \leq n \leq \tau(i)}\right),
$$

and this in turn provides us with

$$
\max _{1 \leq n \leq \hat{\tau}(i)} \hat{S}_{n}^{-} \stackrel{\mathrm{D}}{=} \max _{1 \leq n \leq \tau(i)}\left(S_{\tau(i)}-S_{n}\right)^{-}\left\{\begin{array}{l}
\leq D^{i}+S_{\tau(i)}^{-} \\
\geq D^{i}-S_{\tau(i)}^{-}
\end{array} .\right.
$$

Now, if (d) holds then $\left(S_{\tau_{n}(i)}\right)_{n \geq 0}$ for any fixed $i \in \&$ must be positive divergent. Also,

$$
\mathbb{E}_{i} J_{i}\left(\max _{1 \leq n \leq \hat{\tau}(i)} \hat{S}_{n}^{-}\right)<\infty
$$

by [4, Theorem 6.1]. In combination with (5.2) and (5.3), this yields

$$
\mathbb{E}_{i} J_{i}\left(D^{i}\right) \leq \mathbb{E}_{i} J_{i}\left(\max _{1 \leq n \leq \hat{\tau}(i)} \hat{S}_{n}^{-}\right)+\mathbb{E}_{i} S_{\tau(i)}^{-}<\infty
$$

and (e) follows. Conversely, if (e) and, thus, (5.1) and (5.2) hold, these conditions together with (5.3) imply (5.4) and thence (d) by another appeal to [4, Theorem 6.1]. 
(d) and (f) are equivalent. First, Lemma 2.2 shows directly that (d) implies (f). The converse follows because (f) implies that, for all $i \in \&$,

$$
\mathbb{P}_{i}\left(\liminf _{n \rightarrow \infty} \hat{S}_{n} \geq 0\right)>0
$$

and this in combination with the fluctuation-type trichotomy for nontrivial MRWs gives (d).

(a) implies $(d)$. Consider the Markov-modulated sequence $\left(\left(M_{n}, E_{n}\right), X_{n}\right)_{n \geq 0}$ and recall that $X_{n}+E_{n}-E_{n-1}=S_{n}^{*}-S_{n-1}^{*} \geq 0$ for all $n$. Now, if (a) holds then

$$
\Gamma:=\mathbf{P}_{\Lambda}\left(\left(\left(M_{1}, E_{1}\right), X_{1}\right) \in \cdot\right)
$$

provides us with the stationary distribution of $\left(\left(M_{n}, E_{n}\right), X_{n}\right)_{n \geq 0}$ (which is still a Markov chain). Therefore, we may consider a doubly infinite stationary extension $\left(\left(M_{n}, E_{n}\right), X_{n}\right)_{n \in \mathbb{Z}}$ under a suitable probability measure $\mathbf{Q}$, say. Then

$$
\mathbf{Q}\left(\left(M_{n}, E_{n}\right)_{n \in \mathbb{Z}} \in \cdot\right)=\mathbf{P}_{\Lambda}\left(\left(M_{n}, E_{n}\right)_{n \in \mathbb{Z}} \in \cdot\right)
$$

as well as

$$
\mathbf{Q}\left(\left(M_{n}, X_{n}\right)_{n \in \mathbb{Z}} \in \cdot\right)=\mathbb{P}_{\xi}\left(\left(M_{n}, X_{n}\right)_{n \in \mathbb{Z}} \in \cdot\right)
$$

with $\xi$ as stated in the theorem. Next, use $\left(E_{n}\right)_{n \in \mathbb{Z}}$ together with the doubly infinite extension $\left(S_{n}\right)_{n \in \mathbb{Z}}$ as defined in (2.1) to define the doubly infinite extension $\left(S_{n}^{*}\right)_{n \in \mathbb{Z}}$ in the canonical way, namely, $S_{n}^{*}=S_{n}+E_{n}$ or, equivalently, $S_{n}^{*}-S_{n-1}^{*}=X_{n}+E_{n}-E_{n-1}$ for $n \in \mathbb{Z}$. Then $X_{n}+E_{n}-E_{n-1} \geq 0$ entails $E_{0}=S_{0}+E_{0}=S_{0}^{*} \geq S_{-1}^{*} \geq S_{-2}^{*} \geq \cdots$ and, thus, $\lim \sup _{n \rightarrow \infty} S_{-n} \leq \limsup _{n \rightarrow \infty} S_{-n}^{*} \leq E_{0}<\infty$ Q-a.s. Consequently,

$$
\liminf _{n \rightarrow \infty} \hat{S}_{n}=-\limsup _{n \rightarrow \infty} S_{-n}>-\infty \quad \text { Q-a.s., }
$$

which by another use of the fluctuation-type trichotomy implies (d).

(d) implies (b). Assuming the positive divergence of $\left(\hat{M}_{n}, \hat{S}_{n}\right)_{n \geq 0}$, the doubly infinite sequence of ladder epochs $\left(\sigma_{n}\right)_{n \in \mathbb{Z}}$ from Subsection 2.1 and, thus, also the expressions for $\pi_{i}^{>}$in (3.3) are well defined. If we can verify that $\pi^{>}$is a stationary distribution for $M^{>}$, then (b) follows. This final step also shows that (3.4)-(3.7) hold.

First we show the equality of the two expressions for $\pi_{i}^{>}$in (3.3). We have

$$
\begin{aligned}
\mathbb{E}_{\xi}\left(\frac{1}{\sigma_{1}-\sigma_{0}} \mathbf{1}_{\left\{M_{\sigma_{0}}=i\right\}}\right) & =\sum_{k \geq 0} \sum_{l>k} \frac{1}{l} \mathbb{P}_{\xi}\left(M_{-k}=i, \sigma_{0}=-k, \sigma_{1}-\sigma_{0}=l\right) \\
& =\sum_{k \geq 0} \sum_{l>k} \frac{1}{l} \mathbb{P}_{\xi}\left(M_{0}=i, \sigma_{0}=0, \sigma_{1}-\sigma_{0}=l\right) \\
& =\sum_{l \geq 1} \mathbb{P}_{\xi}\left(M_{0}=i, \sigma_{0}=0, \sigma_{1}-\sigma_{0}=l\right) \sum_{k=0}^{l-1} \frac{1}{l} \\
& =\sum_{l \geq 1} \mathbb{P}_{\xi}\left(M_{0}=i, \sigma_{0}=0, \sigma_{1}-\sigma_{0}=l\right) \\
& =\mathbb{P}_{\xi}\left(M_{0}=i, \sigma_{0}=0\right)
\end{aligned}
$$

where the third line follows from the stationarity of $\left(M_{n}, X_{n}\right)_{n \in \mathbb{Z}}$ under $\mathbb{P}_{\xi}$. This stationarity 
also provides us with

$$
\begin{aligned}
\left\{M_{-k}\right. & \left.=i, \sigma_{0}=-k, \sigma_{1}-\sigma_{0}=l\right\} \\
& =\left\{M_{-k}=i, S_{-k}>\max _{j>k} S_{-j}, \max _{1 \leq j<l} S_{-k+j} \leq S_{-k}, S_{-k+l}>\max _{0 \leq j<l} S_{-k+j}\right\} \\
& =\left\{M_{-k}=i, \min _{j>k} \sum_{n=-j}^{-k} X_{n}>0, \max _{1 \leq j<l} \sum_{n=-k+1}^{-k+j} X_{n} \leq 0, \min _{1 \leq j \leq l} \sum_{n=-k+j}^{-k+l} X_{n}>0\right\} \\
& \stackrel{\mathrm{D}}{=}\left\{M_{0}=i, \min _{j>0} \sum_{n=-j}^{0} X_{n}>0, \max _{1 \leq j<l} \sum_{n=1}^{j} X_{n} \leq 0, \min _{1 \leq j \leq l} \sum_{n=j}^{l} X_{n}>0\right\} \\
& =\left\{M_{0}=i, \sigma_{0}=0, \sigma_{1}-\sigma_{0}=l\right\}
\end{aligned}
$$

for all $0 \leq k<l$, where $A \stackrel{\mathrm{D}}{=} B$ means that $\mathbf{1}_{A}$ and $\mathbf{1}_{B}$ have the same law under $\mathbb{P}_{\xi}$, and, thus,

$$
\frac{1}{c l} \mathbb{P}_{\xi}\left(M_{\sigma_{0}}=i, \sigma_{1}-\sigma_{0}=l\right)=\frac{1}{c} \mathbb{P}_{\xi}\left(M_{0}=i, \sigma_{0}=0, \sigma_{1}=l\right) .
$$

Since

$$
\pi_{i}^{>}=\frac{1}{c} \mathbb{P}_{\xi}\left(\sigma_{0}=0 \mid M_{0}=i\right) \mathbb{P}_{\xi}\left(M_{0}=i\right)=\frac{1}{c} \mathbb{P}_{i}\left(\sigma_{0}=0\right) \pi_{i},
$$

we also obtain the asserted form of the $\pi$-density of $\pi^{>}$.

Concerning the stationarity of $\pi^{>}$, we obtain by a similar argument that, for all $j \in \delta$,

$$
\begin{aligned}
\mathbb{P}_{\pi^{>}}\left(M_{1}^{>}=j\right) & =\sum_{i \in \delta} \pi_{i}^{>} \mathbb{P}_{i}\left(M_{1}^{>}=j\right) \\
& =\frac{1}{c} \sum_{i \in s} \mathbb{P}_{\xi}\left(\sigma_{0}=0, M_{0}=i\right) \mathbb{P}_{i}\left(M_{1}^{>}=j\right) \\
& =\frac{1}{c} \sum_{i \in \delta} \sum_{n \geq 1} \mathbb{P}_{\xi}\left(\sigma_{0}=0, \sigma_{1}=n, M_{0}=i, M_{n}=j\right) \\
& =\frac{1}{c} \sum_{i \in \delta} \sum_{n \geq 1} \mathbb{P}_{\xi}\left(\sigma_{-1}=-n, \sigma_{0}=0, M_{-n}=i, M_{0}=j\right) \\
& =\frac{1}{c} \sum_{i \in s} \mathbb{P}_{\xi}\left(M_{-1}^{>}=i, \sigma_{0}=0, M_{0}=j\right) \\
& =\frac{1}{c} \mathbb{P}_{\xi}\left(\sigma_{0}=0, M_{0}=j\right)=\pi_{j}^{>}
\end{aligned}
$$

and this is the desired result.

Equation (3.4) now follows from the relation that, for all $i \in \delta$,

$$
\begin{aligned}
\mathbb{P}_{\xi}\left(M_{0}=i, \sigma_{0}=0\right) & =\mathbb{P}_{\xi}\left(M_{0}=i, \max _{n \geq 1} S_{-n}<0=S_{0}\right) \\
& =\mathbb{P}_{\xi}\left(\hat{M}_{0}=i, \min _{n \geq 1} \hat{S}_{n}>0\right) \\
& =\pi_{i} \mathbb{P}_{i}\left(\hat{\sigma}_{1}^{\leqslant}=\infty\right) .
\end{aligned}
$$


Using (5.5) and the obvious fact that $\left(M_{n}^{>}, \sigma_{n+1}^{>}-\sigma_{n}^{>}\right)_{n \geq 0}$ is a stationary Markov chain under $\mathbb{P}_{\pi^{>}}$, we obtain for its stationary law $v$ the relation, for all $(i, m) \in \delta \times \mathbb{N}$,

$$
\begin{aligned}
v_{i, m} & =\mathbb{P}_{\pi>}\left(M_{0}^{>}=i, \sigma_{1}^{>}=m\right) \\
& =\pi_{i}^{>} \mathbb{P}_{i}\left(\sigma_{1}^{>}=m\right) \\
& =\frac{1}{c} \mathbb{P}_{\xi}\left(M_{0}=i, \sigma_{0}=0\right) \mathbb{P}_{i}\left(\sigma_{1}^{>}=m\right) \\
& =\frac{1}{c} \mathbb{P}_{\xi}\left(M_{0}=i, \sigma_{0}=0, \sigma_{1}=m\right) \\
& =\frac{1}{c m} \mathbb{P}_{\xi}\left(M_{\sigma_{0}}=i, \sigma_{1}-\sigma_{0}=m\right),
\end{aligned}
$$

and this proves (3.5). Equations (3.6) and (3.7) are now direct consequences, and so (d) implies (b).

Remark 5.1. Assuming that Theorem 3.2(d) holds, the stationarity of $\pi^{>}$defined by (3.3) may also be derived within the framework of Palm calculus as laid out in [19, Chapter 8]. For a short description, we start with the stationary sequence $\left(M_{n}, X_{\leqslant n}\right)_{n \in \mathbb{Z}}$ under $\mathbb{P}_{\xi}, X_{\leqslant n}:=\left(X_{k}\right)_{k \leq n}$, and put here

$$
E_{m}:=S_{m}-\max _{k \leq m} S_{k}=\min _{k \leq m} \sum_{j=k+1}^{m} X_{j} \quad \text { for } m \in \mathbb{Z} .
$$

Then $E_{m}$ is a functional of $X_{\leqslant m}$ which equals 0 if and only if $m$ is a strictly ascending ladder epoch of the associated doubly infinite MRW $\left(M_{n}, S_{n}\right)_{n \in \mathbb{Z}}$. In other words, the sequence $S:=\left(\sigma_{n}\right)_{n \in \mathbb{Z}}$ of ladder epochs may be viewed as the sequence of return times to $\& \times\{0\}$ of the stationary sequence $\boldsymbol{Z}:=\left(M_{n}, E_{n}\right)_{n \in \mathbb{Z}}$, and it is a functional of it. For $n \in \mathbb{Z}$, define the two-sided shift

$$
\theta^{n}\left(\left(z_{k}\right)_{k \in \mathbb{Z}},\left(t_{k}\right)_{k \in \mathbb{Z}}\right):=\left(\left(z_{n+k}\right)_{k \in \mathbb{Z}},\left(t_{n, k}\right)_{k \in \mathbb{Z}}\right)
$$

for $n \in \mathbb{Z},\left(z_{k}\right)_{k \in \mathbb{Z}} \in(\& \times(-\infty, 0])^{\mathbb{Z}}$ and strictly increasing sequences $\left(t_{k}\right)_{k \in \mathbb{Z}}$ such that

$$
-\infty \leftarrow \cdots<t_{-1} \leq 0<t_{1}<\cdots \rightarrow \infty
$$

and $\left(t_{n, k}\right)_{k \in \mathbb{Z}}$ equals the sequence $\left(t_{k}+n\right)_{k \in \mathbb{Z}}$ modulo relabelling so as to have $t_{n, 0} \leq 0<t_{n, 1}$ (see also $[19$, p. 251]). Then the above considerations imply that the sequence

$$
\left(\theta^{n}(\boldsymbol{Z}, \boldsymbol{S})\right)_{n \in \mathbb{Z}}
$$

is $\mathbb{P}_{\xi}$-stationary, and the Palm duality theory [19, Theorem 8.4.1] now tells us that its cycles

$$
\left(\sigma_{n+1}-\sigma_{n},\left(M_{k}, E_{k}\right)_{\sigma_{n} \leq k<\sigma_{n+1}}\right), \quad n \in \mathbb{Z},
$$

and the sequence $\left(M_{\sigma_{n}}, \sigma_{n+1}-\sigma_{n}\right)_{n \in \mathbb{Z}}$ in particular are stationary under the probability measure $\mathbb{P}_{\xi}^{0}$, defined by

$$
\mathbb{P}_{\xi}^{0}(\mathrm{~d} x):=\frac{1}{c\left(\sigma_{1}-\sigma_{0}\right)} \mathbb{P}_{\xi}(\mathrm{d} x)
$$

with $c$ as in Theorem 3.2 and satisfying $\mathbb{P}_{\pi}^{0}\left(\sigma_{0}=0\right)=1$. Consequently,

$$
\pi_{i}^{>}=\mathbb{P}_{\xi}^{0}\left(M_{0}=i\right)=\frac{1}{c} \mathbb{E}_{\xi}\left(\frac{1}{\sigma_{1}-\sigma_{0}} \mathbf{1}_{\left\{M_{\sigma_{0}}=i\right\}}\right), \quad i \in \mathcal{s}
$$

is a stationary distribution for the ladder chain as asserted in our theorem. 
Remark 5.2. The stationarity of $\pi^{>}$, when defined by (3.4), follows also from

$$
I-\|G\|=\left(I-\left\|^{\star} G^{\leqslant}\right\|\right)\left(I-\left\|G^{>}\right\|\right),
$$

which in turn is a direct consequence of the Wiener-Hopf factorization (2.5), namely, by multiplying (5.6) from the left by $\pi^{\top}$ (the transpose of $\pi$ ) and observing that $\|G\|=P=$ $\left(p_{i j}\right)_{i, j \in \delta}$ is the transition matrix of $M$, we deduce that

$$
0=\pi^{\top}(I-P)=\pi^{\top}\left(I-\left\|^{\star} G^{\leqslant}\right\|\right)\left(I-\left\|G^{>}\right\|\right) .
$$

By Lemma 2.2, in particular (2.6), and the fact that all $\pi_{i}$ are positive, the nonnegative vector

$$
\pi^{\top}\left(I-\left\|^{\star} G^{\leqslant}\right\|\right)=\left(\pi_{i} \mathbb{P}_{i}\left(\hat{\sigma}_{1}^{\leqslant}=\infty\right)\right)_{i \in \mathcal{S}}
$$

is not identically zero and, thus, a proper solution of the equation $x\left(I-\left\|G^{>}\right\|\right)=0$. After normalization through $c$, it is therefore the stationary distribution of $M^{>}$with transition matrix $\left\|G^{>}\right\|$.

\section{Examples and concluding remarks}

Examples where the ladder chain $\left(M_{n}^{>}\right)_{n \geq 0}$ is positive recurrent with stationary distribution $\pi^{>}$, but $\mathbb{E}_{\pi}>\sigma_{1}^{>}=\infty$, are not difficult to find, and at Examples 6.1-6.3 below we provide three such instances. By Theorem 3.2, this means that (2.2) must fail and, thus, the dual MRW $\left(\hat{M}_{n}, \hat{S}_{n}\right)_{n \geq 0}$ must oscillate.

Example 6.1. Let $\left(M_{n}, S_{n}\right)_{n \geq 0}$ be a nontrivial MRW with positive recurrent driving chain on a finite state space $\&$ and stationary drift 0 . Such a MRW has oscillating embedded RWs $\left(S_{\tau_{n}(i)}\right)_{n \geq 0}$ and so is solidary, as pointed out in Section 2; thus,

$$
\liminf _{n \rightarrow \infty} S_{n}=-\infty \quad \text { and } \quad \limsup _{n \rightarrow \infty} S_{n}=\infty \quad \text { a.s. }
$$

(see also [2, Theorems 2 and 3] or [4]), and the same holds for its dual $\left(\hat{S}_{n}\right)_{n \geq 0}$. Therefore, condition (2.2) fails, yet the ladder chain is necessarily positive recurrent on some $\varnothing \neq s^{>} \subset \delta$ because $\delta$ is finite.

Example 6.2. Consider the situation of the previous example but with infinite $\delta$ and make the additional assumption that, for some unique $s \in \delta$, the $F_{i s}, i \in \delta$, are concentrated on $(0, \infty)$, while all other $F_{i j}$ are concentrated on $(-\infty, 0)$. Since $X_{\sigma_{n}^{>}}$must be positive for each $n$, we find that $M_{n}^{>}=s$ for all $n \geq 1$ and so the ladder chain is trivially positive recurrent on $\delta^{>}=\{s\}$.

Example 6.3. In this particularly simple example, consider the case that $M$ has infinite state space and is independent of $\left(X_{n}\right)_{n \geq 1}$, the latter variables being i.i.d. with mean 0 and positive variance. Then $\left(M_{n}, S_{n}\right)_{n \geq 0}$ as well as its dual are oscillating. On the other hand, the ladder chain $M^{>}$is clearly independent of $\left(S_{\sigma_{n}}\right)_{n \geq 0}$ as well as positive recurrent with the same stationary distribution as $M$.

Example 6.4. Last but not least, observe that a positive recurrent ladder chain with $\mathbb{E}_{\pi}>\sigma_{1}^{>}=$ $\infty$ also occurs if $\left(M_{n}, S_{n}\right)_{n \geq 0}$ is a solidary positive divergent MRW with null recurrent driving chain having stationary measure $\pi$ such that $\pi\left(f^{>}\right)<\infty$.

Taken together, these four examples indicate that a complete characterization of positive recurrence of $\left(M_{n}^{>}\right)_{n \geq 0}$ in the case that $\mathbb{E}_{\pi}>\sigma_{1}^{>}=\infty$ appears to be a more difficult question to answer. 
Finally, one may ask even more ambitiously for extensions of the results presented here to the situation when the driving chain of the given MRW has uncountable state space and is positive Harris recurrent. Supported by what has been done in [1], we do not believe that this level of generalization is intrinsically deeper. However, it comes with a number of measure-theoretic complications and even limitations regarding, for instance, the use of regeneration schemes determined by the return epochs to certain sets, Wiener-Hopf factorization, or fluctuationtheoretic results as those needed for the proof of Theorem 3.2 and developed in [4].

\section{Acknowledgements}

The author is grateful to an anonymous referee for various suggestions that helped improve the presentation of the article. This research was partially supported by the Deutsche Forschungsgemeinschaft (SFB 878).

\section{References}

[1] Alsmeyer, G. (2000). The ladder variables of a Markov random walk. Prob. Math. Statist. 20, 151-168.

[2] Alsmeyer, G. (2001). Recurrence theorems for Markov random walks. Prob. Math. Statist. 21, $123-134$.

[3] Alsmeyer, G. (2014). Quasistochastic matrices and Markov renewal theory. In Celebrating 50 Years of The Applied Probability Trust (J. Appl. Prob. 51A), eds S. Asmussen, P. Jagers, I. Molchanov and L. C. G. Rogers, Applied Probability Trust, Sheffield, pp. 359-376.

[4] Alsmeyer, G. and Buckmann, F. (2018). Fluctuation theory for Markov random walks. J. Theoret. Prob. 31, 2266-2342.

[5] Arjas, E. And Speed, T. P. (1973). Symmetric Wiener-Hopf factorisations in Markov additive processes. Z. Wahrscheinlichkeitsth. 26, 105-118.

[6] ArJas, E. And Speed, T. P. (1973). Topics in Markov additive processes. Math. Scand. 33, 171-192.

[7] Asmussen, S. (1989). Aspects of matrix Wiener-Hopf factorisation in applied probability. Math. Sci. 14, 101116.

[8] Asmussen, S. (2003). Applied Probability and Queues, 2nd edn. Springer, New York.

[9] ÇInlar, E. (1969). Markov renewal theory. Adv. Appl. Prob. 1, 123-187.

[10] ÇInlar, E. (1974/75). Markov renewal theory: a survey. Manag. Sci. 21, 727-752.

[11] EricKson, K. B. (1971). A renewal theorem for distributions on $R^{1}$ without expectation. Bull. Amer. Math. Soc. 77, 406-410.

[12] FuH, C. D. AND LaI, T. L. (1998). Wald's equations, first passage times and moments of ladder variables in Markov random walks. J. Appl. Prob. 35, 566-580.

[13] Korolyuk, V. S. And Turbin, A. F. (1976). Semi-Markov Processes and Their Applications. Izdat. 'Naukova Dumka', Kiev (in Russian).

[14] Lalley, S. P. (1986). Renewal theorem for a class of stationary sequences. Prob. Theory Relat. Fields 72, 195-213.

[15] Meyn, S. And Tweedie, R. L. (2009). Markov Chains and Stochastic Stability, 2nd edn. Cambridge University Press.

[16] Prabhu, N. U., Tang, L. C. And Zhu, Y. (1993). Some new results for the Markov random walk. J. Math. Phys. Sci. 25, 635-663.

[17] Presman, È. L. (1969). Factorization methods, and a boundary value problem for sums of random variables given on a Markov chain. Izv. Akad. Nauk SSSR Ser. Mat. 33, 861-900.

[18] Sigman, K. (1995). Stationary Marked Point Processes: An Intuitive Approach. Chapman \& Hall, New York.

[19] Thorisson, H. (2000). Coupling, Stationarity, and Regeneration. Springer, New York.

\section{GEROLD ALSMEYER, University of Münster}

Institute of Mathematical Stochastics, Department of Mathematics and Computer Science, University of Münster, Orléans-Ring 10, D-48149 Münster, Germany. Email address: gerolda@math.uni-muenster.de 